pouca atenção à máquina.

No retrato da importância da utilização desse instrumento como coleta de dados, os autores, expõe a seguinte frase de Stott, "se eu pudesse contar a história por palavras não teria tido necessidade de arrastar uma câmera fotográfica".

Fazem eco também da sugestão de Hine de que as imagens dizem mais do que as palavras, permitindo aos investigadores compreender e estudar aspectos da vida que não podem ser investigados através de outras abordagens e que necessitam de registro visual.

\section{CONSIDERAÇÕES FINAIS}

BOGDAN \& BIKLEN (1994) consideram importante, que o próprio investigador, registre os dados no campo, fornecendo imagens para uma inspeção intensa a posterior e que procure sinais, sobre as relações e as atividades desejadas do objeto de estudo.

\section{REFERÊNCIAS BIBLIOGRÁFICAS}

01. BOGDAN, R.C; BIKLEN, S.K. Investigação qualitativa em educação. Porto Editora, LTDA, 1994. p.183-93.

02. D'AVILA NETO, M.I. As representações do corpo feminino na sociedade brasileira contemporânea. In: Psicologia \& Práticas Sociais. Instituto de Psicologia da UERJ, v. 2, n. 1. 1994/1995. p. 91-8.
Durante a fase de análise, as revelações podem levar, para muito além do que teria sido esperado, como meio de lembrar e estudar detalhes que podem ser descurados com esta técnica utilizada para coleta de dados. Através do retrato das organizações, da aparência das pessoas, a disposição dos móveis, o conteúdo das prateleiras, entre outros, que passam a ter valor de história, devido ao registro de imagens realizadas.

BOGDAN \& BIKLEN (1994) finalizam com a colocação de que na procura dos investigadores educacionais, pela compreensão, através do registro de imagens, não são respostas, mas ferramentas que fornecem uma razão para juntar-se para discussão e assim chegar-se às respostas.

Diante do exposto, acredita-se que a técnica de filmagem em vídeo-tape, constitui-se poderosa ferramenta de coleta de dados, quando o assunto é analisar a comunicação não verbal. O investigador deve considerar os pressupostos de BOGDAN \& BIKLEN (1994) ao conceber o plano de estudo.

\section{SAWADA, N.O. Dimensão não verbal da} interação enfermeiro-paciente em situação pré-operatória. Ribeirão Preto, 1990. 97p. Dissertação (Mestrado) - Escola de Enfermagem de Ribeirão Preto, Universidade de São Paulo.

\title{
PARTICIPACIÓN DE LA COMUNIDAD EN LA PROBLEMÁTICA DE SALUD. UN MODELO INTERDISCIPLINARIO DE INVESTIGACIÓN ACCIÓN
}

\section{INTRODUCCION}

Las representaciones sociales de la participación comunitaria relacionadas con los problemas de salud de Villa Mercedes, sector del Municipio de Rosario de Lerma, en Salta Argentina, junto con el estudio sobre los contextos, la definición de la situación, la comprensión que le confieren a la acción los diferentes actores involucrados y la interacción para interpretar los procesos y los escenarios en los cuales actúan, constituyeron el eje central de este trabajo.

El interés surgió ante la observación de la ausencia de una verdadera participación de la comunidad en el área de la salud, elemento que al no estar presente condicionaba numerosas irregularidades en las diferentes etapas del proceso de promoción, prevención y protección de la salud.

* Lic.en Nutrición, Auxiliar de Investigación, Jefa de Trabajos Prácticos en Socioantropología

** Lic.en Servicio Social. Magister Scientiae en Metodología de la Investigación Científica y Técnica. Profesor Adjunto del Departamento de Salud Pública. Profesor en Desarrollo de la Comunidad y en Metodología de la Investigación Científica. Directora Proyecto de Investigación. Facultad de Ciencias de Salud. Universidad Nacional de Salta. Argentina 
El avance de procesos macroeconómicos afecta de manera definitiva el quehacer y la realidad de salud en todos los ámbitos, de modo que resulta indispensable subrayar que el contexto de crisis que afecta a la región de manera profunda, obliga a los sectores sociales, en particular al sector salud, a reaccionar ante la misma.

La participación social se comprende contextualizándola dentro del análisis de la crisis y de la relación entre actores sociales que la determinan, de modo que el mismo modelo de análisis se aplica a la salud, explicitando ciertas circunstancias y peculiaridades específicas, siempre dentro del proceso y contextos globales OPS (1993).

Es indispensable el desarrollo de propuestas y de posicionamientos claros desde los sectores sociales para una mejor calidad de vida; con estrategias y lineamientos que conduzcan a la acción.

La participación social en cuanto hace referencia a salud, y viceversa, y ambas en su relación con el desarrollo, reflejan conflictos de intereses entre los actores sociales con posiciones diversas. En consecuencia, el desarrollo y la evaluación desde y para la salud, requieren reconocer y resolver dichas relaciones conflictivas para lograr un beneficio colectivo.

Según Giomi, quien se propone actuar sobre la participación social, lo hace desde una posición que determina la manera en que puede observarla y actuar. En los intercambios de información entre los actores sociales y los investigadores, cada uno estaría incorporando alguna información de otros, cambiando sus síntesis e interpretaciones y quizás corrigiendo algunas de sus distorsiones subjetivas y errores de interpretación. La subjetividad inicial de cada diagnóstico individual podría ir cediendo espacios en beneficio de la construcción de un diagnóstico intersubjetivo, compartido por todos.

Desde la idea de la sesión conjunta de diagnóstico, se intenta realizar una reconstrucción colectiva de la realidad, un encuentro de personas. La realidad no es una, depende de quien la observe, describa e interprete. Esto potencia el valor del diagnóstico colectivo. Las reconstrucciones intersubjetivas, con aportes de distintos actores que participan en la realidad bajo observación, son más ricas que aquellas reconstrucciones distorsionadas por contemplar un solo punto de vista. Hay una menor probabilidad de distorsiones subjetivas extremas, aunque no por ello podemos decir que son verdades completas.

Para los sectores populares, el mundo de la vida cotidiana es objetivado y resignificado en las actividades de todos los días, sus peculiares condiciones de salud y existencia conforman un conjunto de saberes, creencias, valores y cosmovisiones que les son propias, dentro de ellos se encuentran también los relatos de salud y enfermedad.

El propósito de la participación social es modificar las relaciones sociales de modo que no sean posibles ni aceptables la marginación, la postergación y la inequidad; así la participación deja de ser una opción para transformarse en la estrategia constituyente del bienestar, pues no de otra manera es posible ejercer la autodeterminación requerida para acceder a la producción y usufructo de los medios que definen el bienestar y la calidad de vida.

\section{OBJETIVOS}

- Conocer las concepciones de los actores sociales acerca de la salud y la participación de la comunidad.

- Generar una propuesta que surgida desde lo cotidiano de los actores, sirva para integrar efectivamente a la comunidad en la problemática de salud.

\section{METODOLOGÍA}

La investigación se enmarcó dentro de una metodología cuanti-cualitativa, en una primera etapa se realizó un estudio exploratorio descriptivo el cual muestra dos aspectos, uno de regularidades objetivas como estructuras institucionales, las normativas que dieron origen a los programas de salud en ejecución en la zona y otro, que forma parte de este trabajo, de las condiciones socioeconómicas de los actores, lo que creó un conocimiento teórico de la práctica social y de los elementos limitantes de la concreción en la realidad de lo planificado.

El componente cualitativo estuvo dado en gran medida por la construcción de conceptos interrelacionados en su contexto estructural con referencia permanente al sistema total de las relaciones en la cual están insertos, rescatando al agente social que produce las prácticas y a su proceso de producción.

Este análisis llevó a una construcción social de la realidad social, tomando como base la teoría de BOURDIEU (1990), intentando aprehender la lógica puesta en marcha por los agentes sociales que producen sus prácticas, actuando en un tiempo y en un contexto determinado.

Consideraciones éticas: se informó a todos los participantes sobre los objetivos y la metodología del estudio, consintiendo expresamente en participar; teniendo la posibilidad de retirarse del proyecto cuando lo desearan, a este fin firmaron su expreso consentimiento.

La construcción de la teoría exigió un constante 
ir y venir, generando un verdadero trabajo en espiral, que fue reelaborándose en el transcurso de la investigación constituyendo las condiciones mismas de realización los actores, escenarios y procesos. Se desarrollaron conceptos y comprensiones con un diseño flexible; observando los escenarios y las personas en una perspectiva holística y relacional, en el contexto de su pasado y de la situación en la que se hallaban; interactuando de un modo natural, dentro de su marco de referencia, nada se dio por sobreentendido, todo fue tema de reflexión y análisis.

Permaneciendo próximos a la realidad, se puso énfasis en el rigor metodológico, no se buscó la verdad sino las significaciones que de los hechos realizaban los propios actores, de qué manera se ven a sí mismos, a sus acciones y experiencias, reconstruyendo los diferentes campos y habitus en juego BOURDIEU (1990).

Ese ir hacia la gente... construir desde las propias palabras de las personas y de su conducta, fue el modo de encarar el mundo empírico de la realidad social, en un estrecho ajuste de los diferentes contextos con lo que la gente realmente dice y hace. Todo ello determinó unidades de anclaje de distintos niveles de integración y complejidad conformando una compleja matriz de datos (SAMAJA, 1993).

Se privilegió como herramientas metodológicas la observación y las entrevistas en profundidad para captar las vivencias de los sujetos, las que forman parte de sus prácticas y posibilitan la interacción entre investigador e investigado, obteniéndose elementos de un universo simbólico mayor; la expresión de lo latente en particulares encuentros permitió la manifestación de mentalidades grupales, excediendo los estados subjetivos particulares, como expresión de discursos internalizados provenientes de un fondo común de valores, sus habitus.

Como instrumento de validación de la interpretación se aplicó la triangulación de fuentes y datos intra e intermétodo.

\section{RESULTADOS}

El análisis del diagnóstico preliminar de la situación socioeconómica de una muestra de la población puso en evidencia que el número de integrantes por familia, varia entre 4 y 17 , con un promedio de 10 miembros.

Con respecto a la integración familiar, en el $41 \%$ de las familias no existía la figura del padre en el hogar. En lo referente a situación laboral, de los padres que conviven con sus familias, el $55 \%$ se encuentra en condiciones de trabajo temporal, en el campo, en la industria de la zona o como albañiles, jornaleros u operarios, el $22 \%$ desocupado y sólo un $22 \%$ tiene trabajo estable.

Un elevado porcentaje de las madres, $64 \%$, es ama de casa, $17 \%$ trabaja como empleada doméstica, $6 \%$ son operarias de fábrica y el $13 \%$ recibe pensión y/ o jubilación, mostrando que ésta es una población en constante riesgo, sin estabilidad laboral ni fuentes de trabajo, lo que le impide mantener una salud psico-física y social adecuada.

El nivel de instrucción es bajo tanto en los padres, con idéntico porcentaje, $45 \%$, para primario incompleto y completo, como en las madres, donde un $6,3 \%$ no tiene escolaridad, $44 \%$ no terminó la escuela primaria y sólo el $25 \%$ la terminó. Son numerosas las familias con hijos adolescentes o jóvenes sin escolaridad, que se encuentran desocupados y no asisten a la escuela.

En general, las condiciones de higiene y de habitabilidad de las viviendas son precarias. La propia comunidad reconoce como problemas más sentidos la falta o temporalidad del trabajo, ausencia de guarderías infantiles, falta de viviendas y/o temor a perderla, escasos alimentos, falta de poder adquisitivo para cubrir las necesidades mínimas.

Con relación a los problemas de salud, si bien en su totalidad son controlados por el Agente Sanitario de la zona, su nivel de alarma es bajo, asisten irregularmente a los controles, lo que afecta en gran medida la eficacia y eficiencia de los esquemas preventivos y de los tratamientos.

No se observa integración entre los diferentes sectores de la población y las fuerzas políticas; los problemas que padecen la mayor parte de sus integrantes en el contexto de esa realidad social, no son únicos ni pertenecen exclusivamente a esa comunidad, es evidente además, que están sufriendo un proceso de empobrecimiento.

La pobreza de amplios sectores es marcada, creciendo cada vez más el de pobres urbanos, con bajos ingresos, ocupación inestable o desocupación, sin acceso a servicios de seguridad social, como obras sociales, jubilación, que no les permiten satisfacer adecuadamente sus necesidades básicas.

\section{EL TRABAJO CON LA COMUNIDAD}

El equipo interdisciplinario de investigación integrado mayoritariamente por Licenciadas en Nutrición y en Enfermería, alumnos de ambas carreras y con la participación de profesionales de Ciencias Sociales, todos de la Facultad de Ciencias de la Salud de la Universidad Nacional de Salta, convocó a los 
vecinos de Villa Mercedes y barrios cercanos a reflexionar sobre sus necesidades más sentidas y la importancia de unirse para lograr solucionar problemas comunes de salud. Se trabajó junto con ellos en talleres de Diagnóstico Participativo.

El diagnóstico se basó en el principio de comprender para recién resolver. exigió que se desglosaran numerosos elementos que a veces parecían hasta superficiales, ampliando el conocimiento que se tenia de los mismos para poder con algunas acciones, modificar la situación.

Este diagnóstico participativo exigió dos actividades básicas: recoger información y reflexionar para la toma de decisiones; fueron numerosas las reuniones con personas del lugar, las entrevistas y las visitas, las lecturas de registros y el trabajo de la gente para recoger los datos para conocer mejor los problemas y establecer las prioridades.

En ese momento se puso en evidencia la crítica situación de pobreza y las necesidades de numerosa familias que se ven obligadas a mendigar por la falta de fuentes de trabajo, lo que traía aparejado graves problemas de salud, falta de alimentación y enfermedades.

Evaluadas las prioridades, surgió la acción participante a partir de los problemas detectados por el mismo grupo, estableciendo como punto de partida el encarar la "Reorganización y reapertura del comedor infantil con la participación de la comunidad beneficiaria"; esto planteaba enfrentar hechos irregulares que exigían cambiarse o resolverse con acciones prácticas para encontrar soluciones no sólo a problemas del funcionamiento y de la organización, sino también lograr la participación de la comunidad en la problemática de salud.

Se reflexionó y discutió sobre el problema a resolver, acerca de su desarrollo, origen, relaciones, consecuencias; por cierto que no se lograron resultados de la noche a la mañana; casi siempre se iba y volvía desde hechos concretos de su realidad, reflexionando desde lo que se encontraba a primera vista, hacia una comprensión cada vez más profunda y esclarecedora, relacionándolo con las situaciones más generales y la estructura de la sociedad de la cual forman parte.

La construcción teórica sobre el problema nunca fue acabada y completa en tanto la realidad es compleja y cambiante, reafirmando la concepción de que no se puede en trabajo comunitario, diagnosticar de una vez, sino parcialmente según las circunstancias concretas de la organización, capacidades, tiempo disponible, grado de interés, conciencia de los actores.

De las acciones iniciadas con posterioridad al primer diagnóstico participativo, surgieron otros problemas y otras necesidades de seguir profundizando en la realidad, emergieron nuevas demandas; lo que llevó a instalar el diagnóstico como una actividad permanente. Mediante varios diagnósticos parciales, la comunidad llegó a una mayor comprensión de la complejidad de su realidad, iniciando un proceso de toma de decisiones colectivas y de realización de acciones prácticas para la superación de algunos problemas a partir de la autogestión.

Un grupo de voluntarias organizó la acción concreta planificando y poniendo en marcha el proyecto, estableciendo como condición que las familias beneficiarias deberían ser las que trabajaran y/o colaboraran para mantener el funcionamiento del comedor, que sólo contaban para llevarlo adelante, con lo recaudado por ese grupo y el esfuerzo de las colaboradoras, comenzaba a gestarse un nuevo campo de juego en el que se establecían posiciones, relaciones entre posiciones, intereses, espacios sociales.

Como agente comprometido en los intereses fundamentales de su existencia, el grupo no estaba seguro acerca de la continuidad de la participación de las madres de los beneficiarios, ni de la asistencia alimentaria que debían brindar desde el comedor, reconociendo que en su relación con otros campos, no contaban regularmente, debido a intereses políticos antagónicos, con recursos financieros de otras instituciones, ante ello y como afirmación de su estructura, definió sus relaciones con los agentes e instituciones conformando la comisión permanente del comedor, iniciando paulatinamente el establecimiento de vínculos institucionales.

La organización lograda, soportó conflictos internos, falta de colaboración, insuficiencia de recursos, desconocimiento de diversos aspectos; aún así logró mantenerse y hasta crecer por la buena voluntad, disposición y fuerza del pequeño grupo de colaboradoras responsables, por el grupo de madres trabajadoras y por algunas donaciones recibidas de la Iglesia, de una empresa local, de la Municipalidad y del grupo de catequesis de la zona.

Las exigencias básicas, reglas de funcionamiento, establecidas por el mismo grupo, fueron que las madres debían hacerse cargo de las actividades propias del comedor, que los niños mayores de 10 años debían colaborar con la limpieza y el cuidado de los niños menores, como así también que todos los beneficiarios debían colaborar en la recaudación de fondos (venta de rifas, lotas, etc.)

\section{MODOS DE GESTION CONSTRUIDOS}

En la actualidad se reconoce en la comisión 
directiva del comedor infantil, la intención de modificar los habitus es decir, las formas de actuar, percibir, valorar, sentir y pensar, que han sido internalizados en el curso de su historia, es así que buscaron esclarecer los problemas, sus causas y efectos, relacionaron la mala salud y la mala alimentación con la falta de educación y concientización de las familias, con la disponibilidad de trabajo y apoyo institucional y con la falta de participación consciente y deliberada como esquemas generadores y organizadores tanto de esas prácticas sociales, de sus percepciones y apreciaciones y de las prácticas de los demás agentes.

Diagnosticar participativamente, su realidad concreta resultó vital para su proceso organizativo, por cierto siempre conflictivo. La organización al ir reconstruyendo su realidad sustentándose en conocimientos de la misma, según su propia vivencia desde lo cotidiano, planteó estrategias para un accionar más acertado y resultó una experiencia de amplia participación, más evidente en los líderes, donde se plantearon los problemas sentidos.

Los actores una vez que identificaron sus conflictos plantearon algunas alternativas de solución, continuando su proceso de participación e interrelación interna y externa, con actividades más específicas y de vinculación como la presentación o socialización del diagnóstico en la que además de la propia comunidad, participaron representantes de la Municipalidad, de la Iglesia y profesionales de la UNSa. En esa oportunidad realizaron un pedido de reconocimiento del comedor infantil reabierto, de apoyo financiero, de asesoramiento y de capacitación técnica.

Como verbalización del sentir común la Sra. Angélica Martinez, responsable del grupo, dirigiéndose al Intendente Municipal y a la Asesora de la Dirección de Acción Social de la Municipalidad expresó:

"Queremos ser escuchadas... y que aquí y ante todos nos den su palabra de cumplir con lo que prometen".

Expresión que resumía su sensación de sentimiento de abandono y marginación padecido por éste y otros tantos grupos residentes en las periferias de las grandes ciudades. Su intervención posibilitó el compromiso municipal de apoyar con cajas de alimentos del programa PRANI y provisión de algunos víveres para el comedor. En su intención de mejorar la capacitación a todo nivel, demandaron el dictado de cursos de capacitación en cocina, costura, armado de alpargatas, recreación para los niños.

Del grupo de responsables del comedor surgió la necesidad de desarrollar un proceso socio educativo concientizador con las madres, programando cursos sobre cuidados de salud y de nutrición, determinando participativamente temas referidos a enfermedades y accidentes de la infancia más frecuentes, e incentivación de la participación de la comunidad beneficiaria. A este fin articularon acciones con docentes de la Facultad, organizando talleres de capacitación que se realizaron en el propio comedor.

Los talleres desarrollaron técnicas lúdicas y de dinámica grupal; con metodología participativa, constructivista, reflexiva, creativa e ilustrativa, fueron realizados sobre dos ejes temáticos centrales, la participación comunitaria y los problemas de salud, en particular la desnutrición, los talleres permitieron conocer cuáles eran los conceptos individuales y grupales de los temas tratados, sus opiniones y concepciones acerca de ellos. Los resultados si bien fueron satisfactorios, la asistencia a pesar de la buena disposición inicial, fue menor a la esperada.

Los conceptos internalizados por la mayoría de las madres, por ejemplo, acerca de la desnutrición, expresaban que:

"es la falta de alimentos..., es la falta de vitaminas..., no hay crecimiento $y$ desarrollo..., hay enfermedad..., los niños están desganados...".

Ante la afirmación de algunas madres de que la desnutrición se da por falta de comida solamente, construyeron este concepto:

"no sólo falta de comida, también es falta de información, por ejemplo no sabemos que tienen los alimentos y les damos cualquier cosa..., también se da por falta de actividad de los niños..., por falta de cuidados, por que no tenemos tiempo de atender los niños bien... por problemas familiares.., disgustos, peleas y los chicos no comen bien..., por falta de dinero, comemos lo que podemos...".

Entre todas fueron conformando las causas y/o consecuencias de la enfermedad y de la 
desnutrición, sin tener demasiado en claro al comienzo, la situación, afirmando que la desnutrición era un problema casi exclusivo de los niños. Al trabajar el qué las motivaba a participar en estos talleres, sus respuestas fueron:

"tener más información acerca de la enfermedad, de la desnutrición, conocer las causas, como ayudar a sus hijos con problemas a salir..., saber preparar y cuidar la alimentación de los niños...".

La reflexión sobre la salud, la enfermedad y su problemática fue apoyada con comparaciones, ilustraciones, folletos, desarrollando temas de su interés y elección. Al finalizar los talleres se pudo considerar como internalizada esta problemática de la desnutrición, llegando a considerar la salud como un bien necesario de cuidar, preservar y reclamar. Las reflexiones llevaron su tiempo, ninguno de los logros vino prefabricado y listo, todo necesitó ser trabajado y retrabajado con constancia y compromiso.

Por los resultados obtenidos se señala que la metodología empleada dio cuenta que: la acción pedagógica fue acorde a las características de la población y el acceso al conocimiento fue un aspecto considerado valioso para el cuidado y preservación de la salud. Hubo apropiación del conocimiento, se constató modificación de hábitos alimentarios en el comedor, reforzados en forma individual, notándose en el Centro de Salud, un aumento de controles tanto de salud como antropométricos, organizando por ejemplo "el mes del control antropométrico" una iniciativa de la misma gente y gestionados por una comisión designada para tal fin.

Al discutir sobre como lograr la participación comunitaria, se distinguieron las siguientes construcciones:

"Cuando sentimos que tenemos que participar, nos asustamos..., tendremos que hablar en grupo..., podremos asumir los compromisos? ..., valdrá la pena el esfuerzo?..., sin embargo, al participar podremos expresar nuestras ideas, experiencias, conocimientos ..., Es una forma de hacer valer nuestros derechos y los de nuestra comunidad...".

... "A veces uno tiene ideas, cuesta expresarlas en la forma que uno quiere.., cómo podríamos trabajar, cómo hacerlo mejor ... y que los demás estén de acuerdo..., uno solo no sabe como hacerlo es un problema de comunicación sobre todo...".

"Nosotros tenemos una pequeña organización..., unas cocinan, otras para organizar y hacer de todo..., estamos trabajando, no sabemos si lo estamos haciendo bien..., pero, tratamos de mejorar...".

Cuando se reflexionó sobre cómo podemos participar, respondieron:

"Es tirar para adelante todas juntas..., si nos enseñan y ayudan pienso que voy a seguir para adelante..., aunque sea despacio tengo que hacer algo por los demás, aunque yo sepa poco".

Al analizar como temas de discusión, la aseveración: "Podemos participar dando nuestra opinión, apoyando y realizando actividades, haciendo valer nuestros derechos" y ... "el participar tiene como ventaja el unir esfuerzos, distribuir las tareas, buscar soluciones, valorar y querer nuestra comunidad...", Reflexionaban:

"y ... no es fácil, porque nosotras no sabemos mucho y somos pocas las mamás que nos unimos..., casi nunca nos apoyan, ni la gente ni nadie de otra parte..., no sabemos si lo hacemos bien..., de la Municipalidad o de la Iglesia nos piden cosas..., a veces no sabemos como hacerlas y si están bien ... tenemos miedos...".

Al reconocer que los talleres sobre participación eran propios y de que tenían poder por ser un grupo autogestionario, con el transcurso de los días, perdieron un poco los miedos y fueron trabajando aspectos más específicos.

Los resultados obtenidos fueron buenos, la comisión del comedor trabajó hasta lograr una cierta coordinación con la Municipalidad, cumpliendo con trámites y documentación, con planillas de control de gestión construidas por ellas mismas. La autogestión tan esperada comenzó a nacer.

\section{CONCLUSIONES}

- La conceptualización de la participación a nivel institucional y comunitario, al inicio de la investigación sólo llegaba a ser considerada como una simple 
colaboración de los actores en actividades para el funcionamiento de los comedores; luego del proceso del diagnóstico participativo, mejoraron su capacidad para impulsarla involucrando a nuevos actores a los escenarios de negociación y conciliación.

- Se evidenció en la formulación teórica de programas institucionales, que la participación comunitaria es considerada en toda su amplitud y en todas sus áreas, pero sólo a nivel de ejecución, sea municipal o provincial, perdiendo su sentido, al no estar presentes o considerados los recursos técnicos y financieros para hacerla realidad.

- En general son pocos los que actúan con representatividad y otros pocos sólo reducen su participación a una simple colaboración en actividades, sin intervención en la toma de decisiones ni en la evaluación de proyectos. Los grupos actúan en representación de sus intereses con el propósito de concretizarlos y para ello, en muchos casos, los actores sociales adolecen de ciertos recursos de poder, que definen en gran medida, su capacidad y oportunidad para realizarlos.

\section{LA ESTRATÉGIA DE TALLER Y LA CAPACITACIÓN EN DIAGNÓSTICO PARTICIPATIVO, PERMITIÓ}

- Revalorizar la participación de la comunidad como

\section{REFERENCIAS BIBLIOGRAFICAS}

01. BOURDIEU, P. Sociología y cultura. Grijaldo, México: Grijaldo, 1990. gestor en la solución de los problemas de salud, como en cualquier otro tipo de problema social, constituyó una estrategia que potenció la transformación de las relaciones sociales, desde la dinámica de su propio campo, constituyéndose en un instrumento de conocimiento, que suscitó y motivó a la acción produciendo y proyectando cambios hacia las tendencias, procesos y estrategias del orden comunitario y municipal.

- Los líderes comenzaron a ver a la participación, como a una estrategia constituyente de su propio liderazgo y una manera posible de ejercer la autodeterminación requerida, para acceder a la producción y usufructo de los medios que definen su bienestar y de ese modo establecer relaciones que determinan quienes toman las decisiones, de que manera y respecto de que ámbitos de su existencia.

\section{RECOMENDACIÓN}

- Los programas destinados a la promoción, prevención y protección de la salud, para lograr alcanzar en toda su extensión los objetivos que se propongan, deben establecer para su ejecución la efectiva implementación de diagnósticos participativos y de talleres de reflexión de la propia comunidad involucrada, con la asistencia de equipos interdisciplinarios debidamente capacitados en estas estrategias.

02. OPS/OMS. Desarrollo y fortalecimiento de los sistemas locales de salud. La participación social en el desarrollo social de la salud. Washington, D.C., 1993. (HSS/SILOS - 26).

04. SAMAJA, J. Epistemología y metodología. Buenos Aires: Eudeba, 1993. p. 166-168. 